\title{
Anderson Localization for the Almost Mathieu Equation: A Nonperturbative Proof
}

\author{
Svetlana Ya. Jitomirskaya ${ }^{\star}$ \\ Department of Mathematics, University of California, Irvine, California 92717, USA
}

Received: 10 June 1993

Abstract: We prove that for any diophantine rotation angle $\omega$ and a.e. phase $\theta$ the almost Mathieu operator $(H(\theta) \Psi)_{n}=\Psi_{n-1}+\Psi_{n+1}+\lambda \cos (2 \pi(\theta+n \omega)) \Psi_{n}$ has pure point spectrum with exponentially decaying eigenfunctions for $\lambda \geqq 15$. We also prove the existence of some pure point spectrum for any $\lambda \geqq 5.4$.

\section{Introduction}

In this paper we study localization for the almost-Mathieu operator on $\ell^{2}(\mathbf{Z})$ :

$$
(H(\theta) \Psi)_{n}=\Psi_{n-1}+\Psi_{n+1}+\lambda \cos (2 \pi(\theta+n \omega)) \Psi_{n} .
$$

The almost-Mathieu operator attracted a lot of interest especially in the last decade. For references before 1985 see [1]. Some of the later references are [2-7].

While it is very well understood and commonly believed that for diophantine $\omega$ and $|\lambda|>2$ the operator $H(\theta)$ should have pure point spectrum with exponentially decaying eigenfunctions for almost every $\theta$, this is not yet rigorously proved. Localization was proved by Sinai [2] and Fröhlich, Spencer and Wittwer [3] in the perturbative regime: $|\lambda|$ "big enough." The methods developed in [2] and [3] are very different but have to overcome one common difficulty: the absence of a Wegner-type estimate that would give control of eigenvalue splitting. In both [2] and [3] the gaps in the spectrum were estimated by special inductive multi-scale procedures and these were the hardest parts of the proofs. The estimation of the gaps is in fact the main difficulty in the proof of localization for any potential that is not random enough to be treated by a Wegner-type argument. In this paper we present a new approach to this difficulty: avoiding rather than fighting it. That makes the proof of localization shorter and more elementary.

\footnotetext{
${ }^{\star}$ Permanent address: International Institute of Earthquake Prediction Theory and Mathematical Geophvsics. Moscow. Russia
} 
The proof presented uses many basic ideas of the one in [3], but it is neither a perturbative nor a multiscale type of argument. Namely just one big enough scale is employed. It allows extension of the proof to the range of "not so big" values of $\lambda$ (from now on we assume without loss of generality that $\lambda \geqq 0$ ). The argument as it is works for $\lambda \geqq 15$, but we believe it should be extendible for smaller $\lambda$, maybe even up to the critical value $\lambda=2$. The number 15 (which is actually $14.97 \ldots$ ) looks ugly of course and is an artifact of the "short proof." Surprisingly the method also allows us to prove localization in the center of the band for $\lambda \geqq 5.4$ which somehow contradicts the intuition gained in experience with random potentials that localization should be easier to prove near the edges of the spectrum.

There is of course a price to pay: the method loses the explicit control of the appearance of the gaps and, unlike the method of [2], cannot prove the Cantor character of the spectrum.

We will denote the spectrum of $H(\theta)$ by $\sigma$ and the pure point part of the spectrum by $\sigma_{\mathrm{pp}}$. It is well known that for irrational $\omega$ both $\sigma$ and $\sigma_{\mathrm{pp}}$ (understood as the closure of the set of eigenvalues) do not depend on the phase $\theta$ for a.e. $\theta$. But the dependence on the two other parameters, $\lambda$ and $\omega$, is very nontrivial. For $\lambda>2$ and irrational $\omega$ the Lyapunov exponent is positive; that proves the absence of the absolutely continuous part of the spectrum [8,9]. By Aubry duality for $\lambda<2$ there is no pure point spectrum [4], and there was recently great progress [7] in proving that in this case the spectrum is absolutely continuous for any irrational $\omega$. But for $\lambda>2$ the arithmetic nature of $\omega$ starts to play a major role. Despite the positivity of the Lyapunov exponents, for Liouville $\omega$ (abnormally well approximated by rationals) the spectrum of $H(\theta)$ is purely singular continuous. Anderson localization (pure point spectrum with exponentially decaying eigenfunctions) is expected (and proved for $\lambda$ large) only for "typical" (diophantine or may be satisfying a slightly weaker property) values of $\omega$.

We say that an irrational number $\omega$ is diophantine if there exist $r>1$ and $C>0$ such that

$$
|q \omega-p|>\frac{C}{q^{r}}
$$

for any $p, q \in \mathbf{N}$. Throughout this paper $\omega$ will be assumed to be diophantine.

We will prove

\section{Theorem 1.}

1. For $\lambda>5.4$ there exists an interval $[-\varepsilon(\lambda), \varepsilon(\lambda)]$ such that $\sigma_{\mathrm{pp}} \cap[-\varepsilon(\lambda)$, $\varepsilon(\lambda)] \neq \emptyset$ and the spectrum of $H(\theta)$ in this interval is (for a.e. $\theta$ ) pure point with exponentially decaying eigenfunctions.

2. For $\lambda \geqq 15$ we have $\varepsilon(\lambda) \geqq \lambda+2$ which means Anderson localization for a.e. $\theta$.

Remarks.

1. The function $\varepsilon(\lambda)$ is montone increasing in $\lambda$ and so is the function $\varepsilon(\lambda)-\lambda$. For example $\varepsilon(6) \geqq 4, \varepsilon(9) \geqq 9$ and $\varepsilon(14) \geqq 15$.

2. As can be seen from the proof, the set of $\theta$ 's for which we prove localization, is given by an explicit condition (e.g., $\theta=0$ belongs to this set). Thus Theorem 1 provides a lot of "concrete" examples of operators with pure point spectrum.

3. With only minor changes in the argument (mainly in the proof of the Proposition 5) the same result can be proved for $\omega$ satisfying a weaker 
condition than (1). Namely, let $g(x)$ be any function such that for any $c>0$ we have $e^{-c x}=o(g(x))$. Then the same result holds for all $\omega$ such that for any $p, q \in \mathbf{N}$ we have $|q \omega-p|>g(q)$.

\section{Proof of Theorem 1.}

Let

$$
M(E, \lambda)=\frac{1}{\sqrt{3}}\left|E+i+\sqrt{(E+i)^{2}-\lambda^{2}}\right|,
$$

where by $\sqrt{(E+i)^{2}-\lambda^{2}}$ we understand the value with positive imaginary part. We set

$$
C(E, \lambda)=\frac{\ln \frac{\lambda}{2}}{\ln M(E, \lambda)}-\frac{3}{4} .
$$

Theorem 1 follows immediately from

Theorem 2. If an interval $I$ is such that $C(E, \lambda)>0$ for all $E \in I$ then the spectrum of $H(\theta)$ in $I$ is pure point and the corresponding eigenfunctions are exponentially decaying.

Proof of Theorem 1. It is easy to see that $C(E, \lambda)$ is monotone decreasing in $|E|$ and increasing in $\lambda$. Since $0 \in \sigma$, (see, e.g. [6]) the first statement now follows from $C(0,5.4)>0$.

For any $\lambda$ we have $\sigma \subset[-\lambda-2, \lambda+2]$. In order to finish the proof of the second statement it remains to notice that $C(\lambda+2, \lambda)$ is monotone increasing in $\lambda$ and that $C(16.97,14.97)>0$.

\section{Proof of Theorem 2}

We will use the notation $G_{\left[x_{2}, x_{2}\right]}(E)$ for the Green's function $(H-E)^{-1}$ of the operator $H(\theta)$ restricted to the interval $\left[x_{1}, x_{2}\right]$ with zero boundary conditions at $x_{1}-1$ and $x_{2}+1$. Let us denote

$$
P_{k}(\theta, E)=\operatorname{det}\left[\left.(H(\theta)-E)\right|_{[0, k-1]} \mid .\right.
$$

Notice that $P_{k}(\theta, E)$ is an even function of the argument $\theta+\frac{k-1}{2} \omega$ and can be written as a polynomial of the degree $k$ in $\cos \left(2 \pi\left(\theta+\frac{k-1}{2} \omega\right)\right)$ :

$$
P_{k}(\theta, E)=\sum_{j=0}^{k} b_{j}(E) \cos ^{j}\left(2 \pi\left(\theta+\frac{k-1}{2} \omega\right)\right) .
$$

To simplify the notation we will sometimes omit the dependence on $E$.

It is easy to see that $b_{k}=2 \lambda^{k}$. We now fix $E \in R ; 1<m_{1}<\frac{\lambda}{2}$. Given $k>0$ let us denote

$$
A_{k}=\left\{x:\left|P_{k}(\theta+x \omega)\right|>m_{1}^{k}\right\}
$$


For any $x_{1}, x_{2}=x_{1}+k-1, x_{1} \leqq y \leqq x_{2}$ we have

$$
\begin{aligned}
& \left|G_{\left[x_{1}, x_{2}\right]}\left(x_{1}, y\right)\right|=\left|\frac{P_{x_{2}-y}(\theta+(y+1) \omega)}{P_{k}\left(\theta+x_{1} \omega\right)}\right|, \\
& \left|G_{\left[x_{1}, x_{2}\right]}\left(y, x_{2}\right)\right|=\left|\frac{P_{y-x_{1}}\left(\theta+x_{1} \omega\right)}{P_{k}\left(\theta+x_{1} \omega\right)}\right| .
\end{aligned}
$$

Proposition 1. For any $\varepsilon>1$ there exists $k(\varepsilon, E)$ such that for $k>k(\varepsilon, E)$ and all $\theta$ we have

$$
\left|P_{k}(\theta, E)\right|<(\varepsilon M(E, \lambda))^{k} \text {. }
$$

Proof. We define

$$
\begin{gathered}
B(\theta, E)=\left(\begin{array}{cc}
E-\lambda \cos 2 \pi \theta & 1 \\
-1 & 0
\end{array}\right), B_{k}(\theta, E)=B(\theta+k \omega, E), \\
M_{k}(\theta, E)=B_{k}(\theta, E) \ldots B_{0}(\theta, E) .
\end{gathered}
$$

For $A=\left(\begin{array}{ll}a & b \\ c & d\end{array}\right)$ we define the norm of $A$ as $\|A\|=\max \left(\sqrt{a^{2}+c^{2}}, \sqrt{b^{2}+d^{2}}\right)$. Let $\|A\|_{\text {op }}$ be the operator norm. It is well known that $\mid P_{k}(\theta, E) \leqq\left\|M_{k}(\theta, E)\right\|_{\text {op }} \leqq$ $\prod_{j=0}^{k}\left\|B_{j}(\theta, E)\right\|_{\text {op. }}$

For any matrix $A$ of the form $\left(\begin{array}{ll}c & 1 \\ -1 & 0\end{array}\right)$ we have $\|A\|_{\mathrm{op}} \leqq \frac{2}{\sqrt{3}}\|A\|$, thus $\left|P_{k}(\theta, E)\right| \leqq\left(\frac{2}{\sqrt{3}}\right)^{k+1} \prod_{j=0}^{k}\left\|B_{j}(\theta, E)\right\|$. Since $\|B(\theta, E)\|$ is a continuous function of $\theta$ we can use strict ergodicity of the rotation by the irrational angle $\omega$ to show that for any $\varepsilon>1$ and $k$ large enough we have

$$
\left|P_{k}(\theta, E)\right| \leqq \varepsilon^{k}\left(\frac{2}{\sqrt{3}}\right)^{k} e^{k \int_{0}^{1} \ln \|B(\theta, E)\| d \theta} .
$$

The last integral can be computed directly and is equal to $\ln \left(\frac{\sqrt{3}}{2} M(E, \lambda)\right)$.

It follows from Proposition 1 and (2) that for $x_{1} \in A_{k}, x_{2}=x_{1}+k-1, k>k(\varepsilon)$, $\frac{1}{m_{1}}<m_{2}<1$ and $y \in\left[x_{1}, x_{2}\right]$ such that

$$
k\left(1-\frac{\ln \left(m_{1} m_{2}\right)}{\ln (\varepsilon M(E, \lambda))}\right)<y-x_{1}<k\left(\frac{\ln \left(m_{1} m_{2}\right)}{\ln (\varepsilon M(E, \lambda))}\right),
$$

we have

$$
\left|G_{\left[x_{1}, x_{2}\right]}\left(y, x_{i}\right)\right|<m_{2}^{k}, \quad i=1,2 .
$$

We will denote $\frac{\ln \left(m_{1} m_{2}\right)}{\ln (\varepsilon M(E, \lambda))}$ by $c_{\lambda, \varepsilon}$.

Definition. $A$ point $y \in \mathbf{Z}$ we be called $\left(m_{2}, k\right)$-regular if there exists an interval $\left[x_{1}, x_{2}\right]$ containing $y$ such that

$$
\left|G_{\left[x_{1}, x_{2}\right]}\left(y, x_{i}\right)\right|<m_{2}^{k} \text {, and } \operatorname{dist}\left(y, x_{i}\right) \leqq k ; i=1,2 .
$$

Otherwise $y$ will be called $\left(m_{2}, k\right)$-singular. 
Proposition 2. Suppose $y \in \mathbf{Z}$ is $\left(m_{2}, k\right)$-singular. Then for any $x$ such that $k\left(1-c_{\lambda, \varepsilon}\right) \leqq$ $y-x \leqq k c_{\lambda, \varepsilon}$ we have that $x$ does not belong to $A_{k}$.

Proposition 2 follows immediately from (3).

The key part of the proof is the following lemma:

Lemma 3. For almost every $\theta$ there exists $k_{1}(\theta)$ such that for $k>k_{1}(\theta)$ if the points $x_{1}, x_{2}$ are such that

1) $x_{i}, x_{i}+1, \ldots, x_{i}+\left[\frac{k+1}{2}\right] \notin A_{k}, i=1,2$,

2) $\operatorname{dist}\left(x_{1}, x_{2}\right)>\left[\frac{k+1}{2}\right]$,

$$
\operatorname{dist}\left(x_{1}, x_{2}\right)>\alpha^{k}
$$

with $\alpha=\alpha\left(m_{1}, \lambda\right)>1$.

The proof of Lemma 3 will be given in the Sect. 4 .

Definition. A formal solution $\Psi_{E}(x)$ of the equation $H(\theta) \Psi_{E}=E \Psi_{E}$ will be called $a$ generalized eigenfunction if $\Psi_{E}(x) \leqq C(1+|x|)$ for some $C=C\left(\Psi_{E}\right)<\infty$.

It is well known that to prove pure point spectrum one only needs to prove that generalized eigenfunctions belong to $l^{2}([10]$; see also $[3,11])$.

Let $E(\theta)$ be a generalized eigenvalue of $H_{\theta}, \Psi(x)$ the corresponding generalized eigenfunction.

Lemma 4. For every $x \in \mathbf{Z}$ such that $\Psi(x) \neq 0$ there exists $k_{0}=k_{0}\left(x, m_{2}, \theta, E\right)$ such that for $k>k_{0}$ the point $x$ is $\left(m_{2}, k\right)$-singular.

Lemma 4 is the same kind of statement as Lemma 3.1 in [3] and so is the proof. We now can finish the proof of the theorem. Suppose $C(\lambda, E)>0$. Then there exist $1<m_{1}<\frac{\lambda}{2}, m_{2}<1$ and $\varepsilon>1$ such that $2 c_{\lambda, \varepsilon}-1>\frac{1}{2}$. Assume without loss of generality that $\Psi(0) \neq 0$. Let $|x|$ be bigger than $\max \left[k(\varepsilon, E), k_{1}(\theta) k_{0}\left(0, m_{2}, \theta, E\right)\right]$. Suppose $x$ is $\left(m_{2},|x|\right)$-singular. Since 0 is $\left(m_{2},|x|\right)$-singular, and $2 c_{\lambda, \varepsilon}-1>\frac{1}{2}$, we obtain using Proposition 2 that the points $x_{1}=x-c_{\lambda, \varepsilon}|x|$ and $x_{2}=-c_{\lambda, \varepsilon}|x|$ satisfy the conditions of Lemma 3 with $k=|x|$ for $|x|$ large enough. Applying Lemma 3 we get that $\operatorname{dist}\left(x_{1}, x_{2}\right)=|x|>\alpha^{|x|}$, which gives a contradiction for $|x|$ large and implies that $x$ is $\left(m_{2},|x|\right)$-regular. Thus we have that there exists an interval $\left[y_{1}, y_{2}\right]$ containing $x$ such that

$$
\left|y_{i}-x\right| \leqq|x|,\left|G_{\left[y_{1}, y_{2}\right]}\left(x, y_{i}\right)\right| \leqq m_{2}^{|x|}, i=1,2 .
$$

We now can use the formula

$$
\Psi(x)=G_{\left[y_{1}, y_{2}\right]}\left(x, y_{1}\right) \Psi\left(y_{1}-1\right)+G_{\left[y_{1}, y_{2}\right]}\left(x, y_{2}\right) \Psi\left(y_{2}+1\right)
$$

to obtain the estimate:

$$
|\Psi(x)| \leqq 2 C(1+2|x|) m_{2}^{|x|}
$$




\section{Proof of Lemma 3}

Let $x_{1}, x_{2}$ be as in Lemma 3. We write:

$$
\begin{gathered}
S_{k}=\left\{\theta:\left|P_{k}(\theta)\right|<m_{1}^{k}\right\}, \\
Q_{k}(x)=\sum_{j=0}^{k-1} b_{j}(E) x^{j}+2 \lambda^{k} x^{k} .
\end{gathered}
$$

We consider $Q_{k}(x)$ as a polynomial on $[-1,1]$.

Let

$$
z_{j}=\left\{\begin{array}{c}
\theta_{i}^{\prime}=\theta+\left(\frac{k-1}{2}+x_{i}\right) \omega \\
\cos \left(2 \pi\left(\theta_{1}^{\prime}+j \omega\right)\right), j=0,1, \ldots,\left[\frac{k+1}{2}\right] \\
\cos \left(2 \pi\left(\theta_{2}^{\prime}+\left(j-\left[\frac{k+1}{2}\right]\right) \omega\right)\right), j=\left[\frac{k+1}{2}\right]+1, \ldots, k .
\end{array}\right.
$$

Since $\theta+\left(x_{i}+j\right) \omega \in S_{k}$ for $i=1,2 ; 0 \leqq j \leqq\left[\frac{k+1}{2}\right]$, we have that

$$
\left|Q_{k}\left(z_{j}\right)\right|<m_{1}^{k}, j=0, \ldots, k .
$$

Let us write $Q(x)$ in the Lagrange interpolation form:

$$
Q_{k}(x)=\sum_{j=0}^{k} Q_{k}\left(z_{j}\right) \prod_{l=0, \ldots, k ; l \neq j} \frac{\left(x-z_{l}\right)}{\left(z_{j}-z_{l}\right)} .
$$

We set

$$
\tilde{Q}_{k}^{(j)}(z)=\prod_{l=0, \ldots, k ; l \neq j}\left(z-z_{l}\right) .
$$

Then we have

$$
Q_{k}(x)=\sum_{j=0}^{k} Q_{k}\left(z_{j}\right) \frac{\widetilde{Q}_{k}^{(j)}(x)}{\widetilde{Q}_{k}^{(j)}\left(z_{j}\right)} .
$$

And, in particular, the right and the left hand sides of (4) have the same coefficient, $2 \lambda^{k}$, with $x^{k}$. Thus

$$
2 \lambda^{k}=\sum_{j=0}^{k} \frac{Q_{k}\left(z_{j}\right)}{\widetilde{Q}_{k}^{(j)}\left(z_{j}\right)} \leqq(k+1) m_{1}^{k} \max _{j=0, \ldots, k} \frac{1}{\widetilde{Q}_{k}^{(j)}\left(z_{j}\right)} .
$$

So there exists $j \in[0, k]$ such that

$$
\left|\widetilde{Q}_{k}^{(j)}\left(z_{j}\right)\right| \leqq \frac{(k+1) m_{1}^{k}}{2 \lambda^{k}} .
$$

Proposition 5. For any $b>2$ there exists $k_{0}$ such that for $k>k_{0}$ and $j \in[0, k]$ we have that $\left|\widetilde{Q}_{k}^{(j)}(z)\right|<a^{k}$, any $a>0$ implies there exists $s \in[0, k], s \neq j$ such that

$$
\left|z-z_{s}\right|<(b a)^{\frac{k}{4}} .
$$


Proof. Suppose $\left|z-z_{s}\right|>(b a)^{\frac{k}{4}}$ for all $s \in[0, k], s \neq j$. We can assume without loss of generality that $j \leqq\left[\frac{k+1}{2}\right]$. We can write

$$
\begin{aligned}
\ln \left|\tilde{Q}_{k}^{(j)}\right|(z) \mid= & \sum_{s=0, \ldots,\left[\frac{k+1}{2}\right] ; s \neq j} \ln \left|\left(z-\cos \left(2 \pi\left(\theta_{1}^{\prime}+s \omega\right)\right)\right)\right| \\
& +\sum_{s=0}^{k-1-\left[\frac{k+1}{2}\right]} \ln \left|\left(z-\cos \left(2 \pi\left(\theta_{2}^{\prime}+s \omega\right)\right)\right)\right| \\
\geqq & 4 \ln (b a)^{\frac{k}{4}}+\sum_{s=0, \ldots,\left[\frac{k+1}{2}\right] ; s \neq j} f_{k}\left(z-\cos \left(2 \pi\left(\theta_{1}^{\prime}+s \omega\right)\right)\right) \\
& +\sum_{s=0, \ldots, k-1-\left[\frac{k+1}{2}\right]} f_{k}\left(z-\cos \left(2 \pi\left(\theta_{2}^{\prime}+s \omega\right)\right)\right),
\end{aligned}
$$

where

$$
f_{k}(z)=\left\{\begin{array}{l}
\ln |z|,|z|>\frac{C_{1}}{k^{2 r}} \\
\ln \frac{C_{1}}{k^{2 r}},|z| \leqq \frac{C_{1}}{k^{2 r}}
\end{array}\right.
$$

$C_{1}=\frac{2^{2 r} C^{2}}{18}, C$ and $r$ are as in (1), since the diophantine property (1) implies that there exist not more than 4 points $s \in[0, k]$ such that $\left|z-z_{s}\right| \leqq C_{1} k^{-2 r}$.

Let $\omega=\left[k_{1}, \ldots, k_{n}, \ldots\right]$ be the continuous fractions expansion of $\omega$; $\frac{p_{n}}{q_{n}}=\left[k_{1}, \ldots, k_{n}\right]$-the $n^{\text {th }}$ approximant. We will use the following properties of the continuous fractions expansions (see, e.g., [12]):

$$
\left|q_{i} \omega-p_{i}\right|<\frac{1}{q_{i+1}}
$$

and

$$
q_{i} \geqq \sqrt{2^{i-1}}
$$

Pick $n$ such that $q_{n} \leqq\left[\frac{k+1}{2}\right]<q_{n+1}$. Inequality (9) implies that $n+1<3 \ln k$ for $k>64$. We can represent $\left[\frac{k+1}{2}\right]$ as $\left[\frac{k+1}{2}\right]=b_{n} q_{n}+\ldots+b_{1} q_{1}+b_{0}$, where for each $0 \leqq j \leqq n$,

$$
b_{j}=\left[\frac{\left[\frac{k+1}{2}\right]-b_{n} q_{n}-\ldots-b_{j+1} q_{j+1}}{q_{j}}\right]
$$




$$
b_{n}=\left[\frac{\left[\frac{k+1}{2}\right]}{q_{n}}\right] .
$$

(8) together with (1) imply that

$$
\begin{gathered}
b_{i}<\frac{q_{i+1}}{q_{i}}<\frac{q_{i+1}}{\left(C q_{i+1}\right)^{1 / r}}<C^{-1 / r}\left[\frac{k+1}{2}\right]^{1-1 / r}, i=0, \ldots, n-1 ; \\
b_{n}<\frac{\left[\frac{k+1}{2}\right]_{n}}{q_{n}}<C^{-1 / r}\left[\frac{k+1}{2}\right]^{1-1 / r} .
\end{gathered}
$$

The right hand side of (7) can be estimated from below as:

$$
\begin{aligned}
& 4 \ln (b a)^{\frac{k}{4}}+(k+1) \int_{0}^{1} f_{k}(z-\cos 2 \pi \theta) d \theta-2\left(b_{n}+\ldots+b_{0}\right) \operatorname{Var} f_{k} \\
& \geqq k \ln (b a)+(k+1) \int_{0}^{1} \ln |z-\cos 2 \pi \theta| d \theta+6 \ln k C^{-1 / r}\left[\frac{k+1}{2}\right]^{1-1 / r} \ln \frac{C_{1}}{k^{2 r}} \\
& \geqq k \ln (b a / 2)+6 C^{-1 / r}\left[\frac{k+1}{2}\right]^{1-1 / r} \ln k \ln \frac{C_{1}}{k^{2 r}}-\ln 2>k \ln a .
\end{aligned}
$$

Here we estimated the error in the ergodic theorem using a standard technique (see, e.g., Lemma 4.1, Ch. 3, [12]). The contradiction proves the statement of the proposition.

Let us fix $\frac{m_{1}}{\lambda}<a<\frac{1}{2}$ and $2<b<\frac{1}{a}$.

Proposition 5 implies that for $k$ large enough there exists $j_{0} \in[0, k], j_{0} \neq j$ such that

$$
\left|z_{j_{0}}-z_{j}\right| \leqq(b a)^{\frac{k}{4}}
$$

which means that there exist $j_{s_{1}}, j_{s_{2}}, j_{s_{t}} \in\left[0,\left[\frac{k+1}{2}\right]\right] \cup\left[x_{2}-x_{1}, x_{2}-x_{1}+k-1-\right.$ $\left.\left[\frac{k+1}{2}\right]\right], i=1,2$, such that

$$
\left|\cos \left(2 \pi\left(\theta_{1}^{\prime}+j_{s_{1}} \omega\right)\right)-\cos \left(2 \pi\left(\theta_{1}^{\prime}+j_{s_{2}} \omega\right)\right)\right| \leqq(b a)^{\frac{k}{4}}
$$

Inequality (10) implies that either

$$
\sin \left(\frac{\left(j_{s_{1}}-j_{s_{2}}\right) \omega}{2}\right) \leqq\left(\frac{(b a)^{\frac{k}{4}}}{2}\right)^{\frac{1}{2}}
$$

or

ii)

$$
\sin \left(\theta_{1}^{\prime}+\frac{\left(j_{s_{1}}+j_{s_{2}}\right) \omega}{2}\right) \leqq\left(\frac{(b a)^{\frac{k}{4}}}{2}\right)^{\frac{1}{2}}
$$

Condition (1) together with (11) implies 


$$
\left|x_{2}-x_{1}\right|>\left|j_{s_{1}}-j_{s_{2}}\right|-k-1>\left(\frac{C}{\left(8(b a)^{\frac{k}{4}}\right)^{\frac{1}{2}}}\right)^{\frac{1}{r}}-k-1>m_{3}^{k}
$$

with $m_{3}=(b a)^{-\frac{1}{9 r}}$, for $k$ large enough. define

The event ii) can occur for a specific set of $\theta$ only. Namely for every $j_{s_{1}}, j_{s_{2}}$ we

$$
\Theta_{j_{S_{1}}, j_{S_{2}}}=\{\theta:(12) \text { holds }\} \text {. }
$$

For every pair $s_{1}, s_{2}$ the set $\Theta_{j_{S_{1}}, j_{S_{2}}}$ is independent of $E$ and has measure not exceeding $\left(32(b a)^{\frac{k}{4}}\right)^{\frac{1}{2}}$. The total number of pairs $s_{1}, s_{2}$ such that $j_{s_{t}} \in\left[0,\left[\frac{k+1}{2}\right]\right] \cup\left[x_{2}-x_{1}, x_{2}-x_{1}+k-1-\left[\frac{k+1}{2}\right]\right], i=1,2$, does not exceed $\frac{1}{4}(k+1)^{2}$. Thus the set

$$
\Theta_{k}=\bigcup_{s_{1}, s_{2}: j_{s_{t}} \in\left[0,\left[\frac{k+1}{2}\right]\right] \cup\left[x_{2}-x_{1}, x_{2}-x_{1}+k-1-\left[\frac{k+1}{2}\right]\right], i=1,2} \Theta_{j_{S_{1}}, j_{S_{2}}}
$$

has measure not exceeding $\frac{1}{4}(k+1)^{2}\left(32(b a)^{\frac{k}{4}}\right)^{\frac{1}{2}}$ and using the Borel-Cantelli lemma we obtain the statement of the lemma.

Acknowledgement. I am deeply grateful to A. Klein for his support and many fruitful discussions.

\section{References}

1. Cycon, H.L., Froese, R.G., Kirsch, W., Simon, B.: Schrödinger operators. Berlin Heidelberg New York: Springer, 1987

2. Sinai, Ya.: Anderson localization for one-dimensional difference Schrödinger operator with quasi-periodic potential. J. Stat. Phys. 46, 861-909 (1987)

3. Fröhlich, J., Spencer, T., Wittwer, P.: Localization for a class of one dimensional quasisperiodic Schrödinger operators. Commun. Math. Phys. 132, 5-25 (1990)

4. Delyon, F.: Absence of localization for the almost Mathieu equation. J. Phys. A 20, L21-L23 (1987)

5. Chulaevski, V., Delyon, F.: Purely absolutely continuous spectrum for almost Mathieu operators. J. Stat. Phys. 55, 1279-1284 (1989)

6. Avron, J., van Mouche, P., Simon, B.: On the measure of the spectrum for the almost Mathieu operator. Commun. Math. Phys. 132, 103-118 (1990)

7. Last, Y.: A relation between absolutely continuous spectrum of ergodic Jacobi matrices and the spectra of periodic approximants. Commun. Math. Phys. 151, 183-192 (1993)

8. Avron, J., Simon, B.: Almost periodic Schrödinger operators. II. The integrated density of states. Duke Math. J. 50, 369-391 (1983)

9. Figotin, A., Pastur, L.: The positivity of Lyapunov exponent and absence of absolutely continuous spectrum for almost Mathieu equation. J. Math. Phys. 25, 774-777 (1984)

10. Fröhlich, J, Martinelli, F., Scoppola, E., Spencer, T.: Constructive proof of localization in the Anderson tight binding model. Commun. Math. Phys. 101, 21-26 (1985)

11. von Dreifus, H., Klein, A.: A new proof of localization in the Anderson tight binding model. Commun. Math Phys. 124, 285-299 (1989)

12. Cornfeld, I., Fomin, S., Sinai, Ya.: Ergodic theory. Berlin Heidelberg New York: Springer, 1982 
